\title{
Diagnostic pitfalls in a young Romanian ranger with an acute psychotic episode
}

\author{
This article was published in the following Dove Press journal: \\ Neuropsychiatric Disease and Treatment \\ 3 May 2016 \\ Number of times this article has been viewed
}

\author{
Előd Ernő Nagy ${ }^{1,2}$ \\ Attila Rácz ${ }^{3}$ \\ Edit Urbán ${ }^{4}$ \\ Gabriella Terhes ${ }^{4}$ \\ Timea Berki ${ }^{5}$ \\ Emőke Horváth ${ }^{6}$ \\ Anca M Georgescu ${ }^{7}$ \\ Iringó E Zaharia-Kézdi ${ }^{7}$ \\ 'Department of Pharmaceutical \\ Biochemistry, University of Medicine \\ and Pharmacy of Târgu-Mureș, \\ ${ }^{2}$ Laboratory of Medical Analysis, \\ Mures Clinical County Hospital, \\ ${ }^{3}$ II. Psychiatry Clinic, Mures Clinical \\ County Hospital, Târgu Mureș, \\ Romania; ${ }^{4}$ Faculty of Medicine, \\ Institute of Clinical Microbiology, \\ University of Szeged, Szeged, ${ }^{5}$ Faculty \\ of Medicine, Institute of Immunology \\ and Biotechnology, University of \\ Pécs, Pécs, Hungary; ${ }^{6}$ Department \\ of Pathology, ${ }^{7}$. Clinic of Infectious \\ Disease, University of Medicine and \\ Pharmacy, Târgu Mureș, Romania
}

\begin{abstract}
The identification and distinction of the pathological conditions underlying acute psychosis are often challenging. We present the case of a 35-year-old ranger who had no history of acute or chronic infectious disease or any previous neuropsychiatric symptoms. He arrived at the Psychiatry Clinic and was admitted as an emergency case, displaying bizarre behavior, hallucinations, paranoid ideation, and delusional faults. These symptoms had first appeared 7 days earlier. An objective examination revealed abnormalities of behavior, anxiety, visual hallucinations, choreiform, and tic-like facial movements. After the administration of neuroleptic and antidepressant treatment, he showed an initial improvement, but on day 10 entered into a severe catatonic state with signs of meningeal irritation and was transferred to the intensive care unit. An electroencephalogram showed diffuse irritative changes, raising the possibility of encephalitis. Taking into consideration the overt occupational risk, Borrelia antibody tests were prescribed and highly positive immunoglobulin (Ig)M and IgG titers were obtained from serum, along with IgG and antibody index positivity in cerebrospinal fluid. In parallel, anti-Nmethyl-D-aspartate receptor antibodies and a whole battery of other autoimmune encephalitis markers showed negative. A complex program of treatment was applied, including antibiotics, beginning with ceftazidime and ciprofloxacin - for suspected aspiration bronchopneumonia - and thereafter with ceftriaxone. A gradual improvement was noticed and the treatment continued at the Infectious Disease Clinic. Finally, the patient was discharged with a doxycycline, antidepressant, and anxiolytic maintenance treatment. On his first and second control (days 44 and 122 from the disease onset), the patient was stable with no major complaints, Borrelia seropositivity was confirmed both for IgM and IgG while the cerebrospinal fluid also showed reactivity for IgG on immunoblot. On the basis of the putative occupational risk, acute psychotic episode, and the success of antibiotic therapy, we registered this case as a late neuroborreliosis with atypical appearance.
\end{abstract}

Keywords: Borrelia burgdorferi, neuroborreliosis, neuropsychiatric symptoms, encephalitis, anti-NMDAR

\section{Introduction}

Neuropsychiatric manifestations characterize the disease course of $\sim 15 \%$ of Borreliainfected individuals. ${ }^{1}$ The involvement of the central nervous system is rare, but encephalitis or encephalomyelitis may appear both in acute and late neuroborreliosis (LNB). ${ }^{2}$ Chronic or LNB as a tertiary disease stage is a well-known entity; however, the underlying pathomechanisms are the subject of intense scientific debate. Supporters of the pathogen persistence theory claim successful cultivation of Borrelia burgdorferi and/or isolation of its DNA from leptomeningeal infiltrates, subpial, or subependimal lesions. ${ }^{1,3}$ Regarding its histological forms, LNB appears either as an infiltrative or an atrophic meningoencephalitis. ${ }^{1}$ Overt psychiatric manifestations
Correspondence: Előd Ernő Nagy Department of Pharmaceutical Biochemistry, University of Medicine and Pharmacy of Târgu-Mureș, 38, Gh. Marinescu St, Târgu-Mureș, 540I39,

Romania

Tel +40 265 2I 555 I 285

Fax +40 265210407

Email elod.nagy@umftgm.ro
Neuropsychiatric Disease and Treatment 2016:12 96I-967

961

Dovepress f 10

http://dx.doi.org/1 0.21 477/NDTS103300 (c) (1) (-) 2016 Nagy et al. This work is published and licensed by Dove Medical Press Limited. The full terms of this license are available at https://www.dovepress.com/terms.php hereby accept the Terms. Non-commercial uses of the work are permitted without any further permission from Dove Medical Press Limited, provided the work is properly attributed. For permission for commercial use of this work, please see paragraphs 4.2 and 5 of our Terms (https://www.dovepress.com/terms.php). 
of LNB as acute psychosis, ${ }^{4-7}$ hallucinations, ${ }^{8}$ paranoia, or obsessive compulsive disorder ${ }^{9-11}$ are not typical, but have been reported in the literature. Sometimes, psychiatric symptoms may be the only evident disorder in neuroborreliosis. Musical hallucinations with sudden onset may appear without hearing loss. ${ }^{8}$ Body schema disturbances, metamorphosia, and auditory hallucinations were described in a 7-year-old boy without any neurological signs. ${ }^{12}$

According to the European Federation of Neurological Societies' guidelines, in order to confirm neuroborreliosis, three criteria (neurological symptoms, cerebrospinal fluid [CSF] pleocytosis, and specific intrathecal antibodies) must be present, and to raise the suspicion of neuroborreliosis, two of these conditions should be present. $^{2}$

The 2013 European Concerted Action on Lyme Borreliosis diagnostic guidelines (www.eucalb.com) determine that the common CSF findings of LNB are intrathecal antibody production and presence of oligoclonal immunoglobulin (Ig)G bands, along with the impairment of the blood-brain barrier.

Here, we present the case of a young adult male patient who was admitted to the Psychiatric Clinic with symptoms of acute psychosis: paranoic delusions, hallucinations, and transient confusional states. He presented advanced symptoms of an encephalitis-like syndrome and catatonia and was sent to the intensive care unit (ICU), where anti-Borrelia serum and central nervous system-specific antibodies were determined. Antibiotic treatment was applied and the patient was transferred to the Infectious Disease Clinic, where his condition slowly began to improve.

Written consent was obtained from the patient to accept the diagnostic and therapeutic procedures, and processing of his medical data for scientific purposes. Approval was received from the Ethics Committee of the Mures Clinical County Hospital (no. 20305/09.12.2015).

\section{Case report}

A previously healthy 35-year-old male was referred to our emergency room following a 7-day history of disturbed behavior, persecutory type paranoid delusions, visual hallucinations, sleeplessness, agitation, and transient confusional states. The family and his employer reported that in this period he could not fulfill his daily tasks, either at his workplace or in the family, and he seemed on two or three occasions "disconnected" from reality for short periods of time. On examination, he presented bizarre behavior, tic-like orofacial movements, choreiform movements of the arms, bradypsychia, persecutory type paranoid delusional thoughts, suspiciousness, visual hallucinations, flat affect, psychomotor anxiety, insomnia, and social withdrawal with poor insight. Additionally, he showed reluctance to stay in hospital for further investigations and treatment.

Neuroleptic (4 $\mathrm{mg}$ risperidonum) and antidepressant (30 mg mirtazapinum) treatment was prescribed. The next day, he became logorrheic and 1,000 mg of sodium valproate was added to the treatment program. Routine laboratory workup for biochemical parameters and complete blood count along with the cranial computed tomography were normal.

On the fourth day of hospitalization, he presented slight muscle rigidity of the upper limbs (then thought to be caused by neuroleptics) for which $6 \mathrm{mg}$ of trihexyphenidyl hydrochloride was added to his treatment.

After a gradual improvement, on the sixth day, the patient presented a short (a few minutes long) transitory confusional state (confusing the medical personnel with his parents).

On the ninth day, he was complaining of dizziness, postural imbalance, and increasing fatigue. A cranial native computed tomography scan showed mild cerebral edema and manitol infusion was administered. The same day, his condition worsened and he entered into a catatonic state. Shortly after, he presented an epileptic seizure. Neurological examination found no focal signs and no pathological reflexes. On the tenth day, the patient re-entered a state of catatonic stupor interrupted with short periods of catatonic agitation. His general condition worsened showing nuchal rigidity, mydriasis, bilateral Babinsky sign, vomiting, and after aspiration of gastric fluid, he was transferred to the ICU with a $92 \%$ oxygen saturation value. Laboratory testing showed a mild elevation of the white blood cell and neutrophil count $\left(11.96 \times 10^{9} / \mathrm{L}\right.$ and $\left.9.68 \times 10^{9} / \mathrm{L}\right)$. Leukocytosis and neutrophilia increased further $\left(15.97 \times 10^{9} / \mathrm{L}\right.$ and $\left.12.55 \times 10^{9} / \mathrm{L}\right)$, being accompanied by monocytosis $\left(2.18 \times 10^{9} / \mathrm{L}\right)$. Neurological examination did not disclose any focal signs but electroencephalogram findings raised the suspicion of encephalitis (Figure 1A-C). The infectious disease specialist could not find any clinical evidence of ongoing infectious disease.

A blood test for Borrelia-specific antibodies was ordered taking into account that the patient was a ranger, although he denied he had had tick-bites or obvious erythema migrans previously. Borrelia antibodies were determined by an automated recombinant antigen mixture-based enzyme-linked immunosorbent assay approach applying MIKROGENs' recomWell Borrelia IgG/IgM diagnostic kits (Neuried, Germany). Both serum IgM and IgG were positive (26.6 and $81.2 \mathrm{U}$, respectively). On the 13 th day in the ICU, a lumbar puncture was performed. The CSF showed slightly elevated glucose $(81 \mathrm{mg} / \mathrm{dL})$, but normal protein level and 


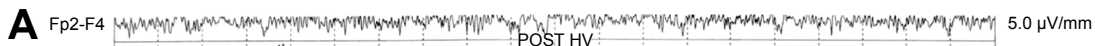

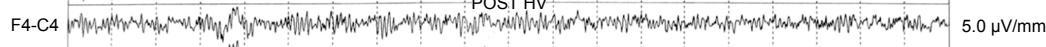

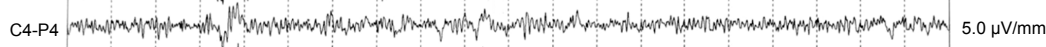

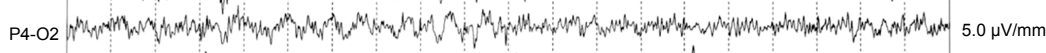

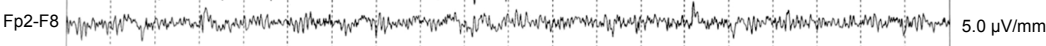

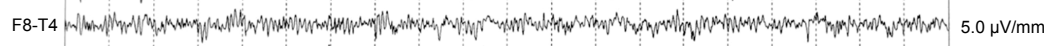

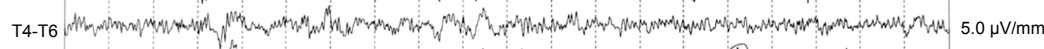

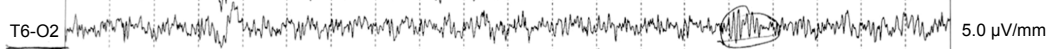

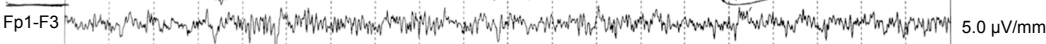

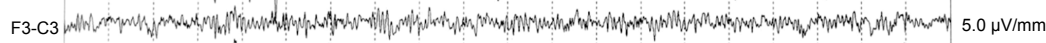

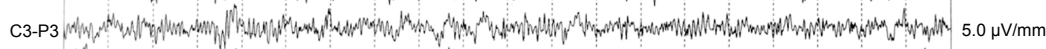

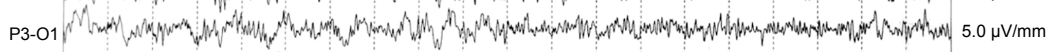

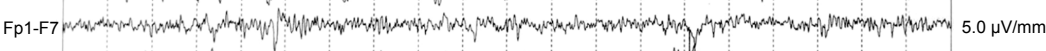

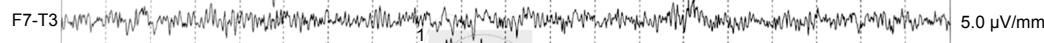

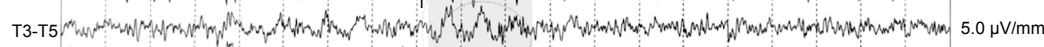

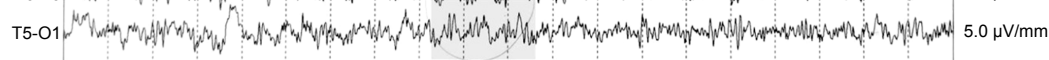
MK-RF

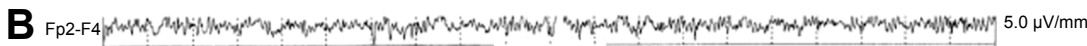

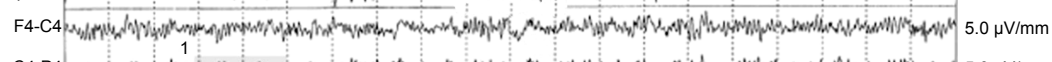

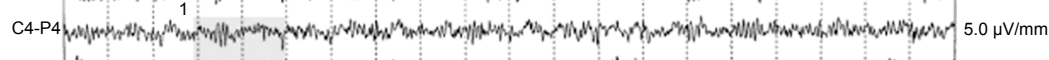

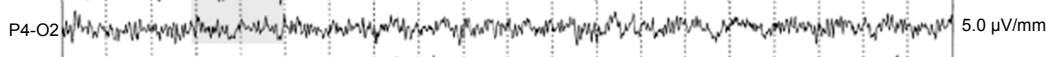

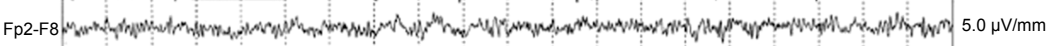

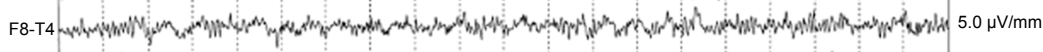

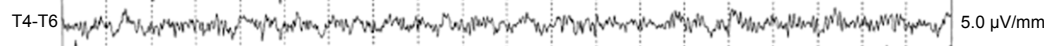

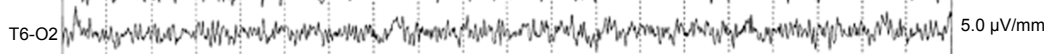

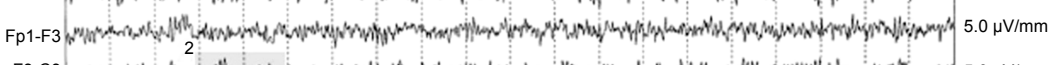

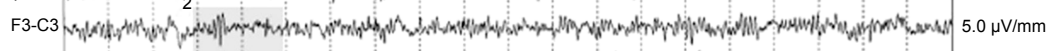

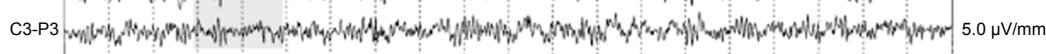

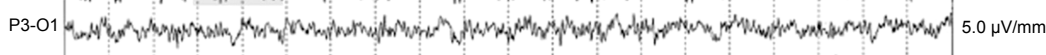

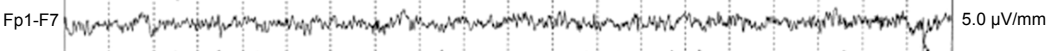

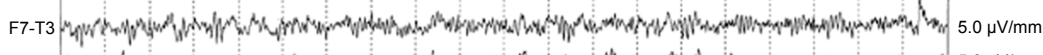

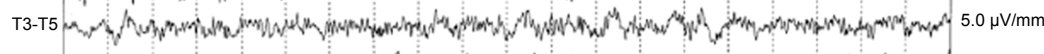

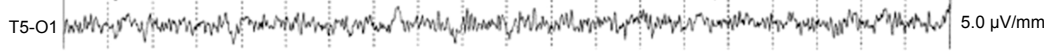

C (+) High pass filter $5.30 \mathrm{~Hz}$ low pass filter $20.0 \mathrm{~Hz}$ EEG reference 50 microvolt/cm notch: yes

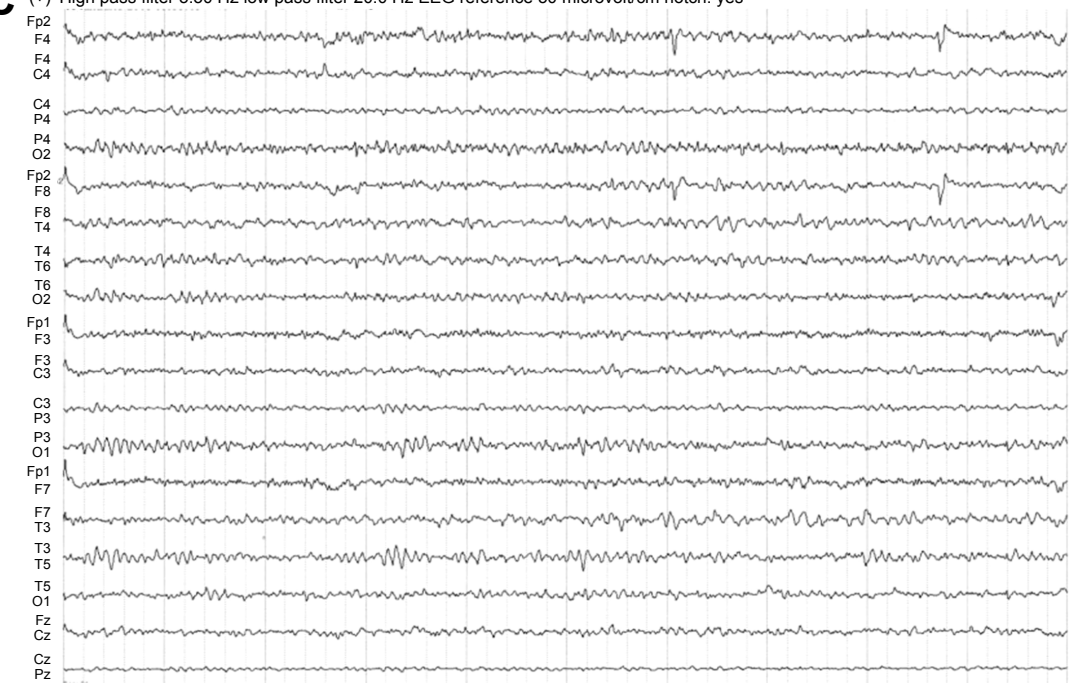

Figure I (A) First EEG pattern of the patient: atypical delta wave complexes on our patient's EEG. Left postero-temporal focal lesion with polymorph delta waves (I - shaded). (B) Second EEG pattern of the patient: alpha-basic rhythm with 8-9 cycles per second and with diffuse irritative changes, unmodified during eye opening, with asymmetric slow sharp wave discharges in the C-T region bilaterally (I and 2 - shaded). (C) Normal EEG pattern for comparison: a basic activity with subalpha-theta waves, without pathological changes and asymmetry. (A and B) Registrations with time constant $0.10 \mathrm{~s}$, high frequency filter $30.0 \mathrm{~Hz}$, notch filter: yes, sensitivity: $5.0 \mu \mathrm{V} / \mathrm{mm}$. (C) Registration with time constant $0.10 \mathrm{~s}$, high pass filter $5.30 \mathrm{~Hz}$, low pass filter $20.0 \mathrm{~Hz}$, notch filter: yes.

Abbreviation: EEG, electroencephalogram. 
LDH activity. CSF presented a few red blood cells, but no pleocytosis, with leucocytes being completely absent.

Paired serum and CSF samples confirmed Borrelia sensu lato serum IgM and IgG positivity (39.7 and 116.2 U) and, also, a positive IgG in CSF (24 U). The antibody index (AI) for IgG was calculated with MIKROGENs' software and found to be positive: 2.83 . Contrast computed tomography scan of the brain performed on the same day was normal.

A day later, immunoblot assay was performed to confirm Borrelia infection by applying Mikrogens' RecomLine Borrelia IgG/IgM kit: this gave a clearly positive result for serum IgM (8 points, with Osp $\mathrm{C}$ reactivities for B. burgdorferi, Borrelia afzelii, Borrelia garinii, and Borrelia spielmanii) and $\operatorname{IgG}$ (25 points, with reactivities for $\mathrm{p} 100$, p58, p39, p41, variable major protein-like sequence (VIsE), and p18) but it was negative for CSF IgM and IgG (tests performed only with high-dilution, 1:20, due to the limited volume of CSF). A 16srRNA PCR was performed on CSF at the Institute of Clinical Microbiology, Szeged, Hungary, and a negative result was obtained. After interdisciplinary consultations, the suspicion of autoimmune encephalitis was raised, and a comprehensive autoantibody test panel was performed for CSF at the Institute of Immunology, University of Pécs (Hungary) including anti-N-methyl-D-aspartate receptor (anti-NMDAR), anti- $\gamma$-aminobutyric acid $B$ receptor (anti-GABA-BR), anti- $\alpha$-amino-3-hydroxy-5-methyl-4 isoxazolepropionic acid receptor 1 (anti-AMPA1) and 2 (AMPA2), anti-leucin-rich glioma-inactivated 1 (anti-LGI1), and anti-contactin-associated protein-like 2. The autoantibody detection method was an indirect immunofluorescence assay (Euroimmun Gmbh, Lübeck, Germany), with antiNMDAR antibodies being also determined from serum. All these autoantibody tests returned negative results.

At this point, the Glasgow Coma Score was 10. Due to suspected aspiration pneumonia, antibiotics were administered (ceftazidime and ciprofloxacin intravenous), and for his psychomotor agitation, haloperidol and diazepam were administered. He was wearing an oxygen mask and was being fed through a nasogastric tube. Glucose $\mathrm{KCl}$ and $\mathrm{NaCl}$ were given for hydroelectrolytic balance. From the 14th day, due to suspected neuroborreliosis, the antibiotic therapy was changed; he received ceftriaxon instead of ceftazidime, while the psychiatric checkup indicated the need for diazepam and the cessation of haloperidol. During his stay in the ICU, he was barely cooperative, afebrile, his blood pressure varied between 130/77 and 160/100 $\mathrm{mmHg}$, and from time to time he showed both respiratory instability and mental confusion. From day 17 , he started to be increasingly cooperative, with an improved general status. Tube feeding was suspended and oral feeding initiated. White blood cell, neutrophil, and monocyte counts decreased gradually to normal values. Serology tests for anti-human immunodeficiency virus 1 and 2, p24 protein, anti-Epstein-Barr virus viral capsid antigen, anti-herpes simplex virus 1 and 2 , and hepatitis $\mathrm{B}$ and $\mathrm{C}$ virus antibodies were all negative. On the 21 st day, he was transferred from the ICU to the Infectious Diseases Clinic I in a conscious, cooperative, hemodynamically, and respiratorily stable state, and showing marked asthenia, adynamia, and fatigue. Biochemical parameters, urinalysis, and erythrocyte sedimentation rate were in their normal ranges. During hospitalization, he continued treatment with ceftriaxon, vitamins from group B, and alprazolam. His general state did not change; he complained of bilateral arm paresthesia.

After 10 days of supportive treatment, on the 32nd day from admission, he was discharged with the following recommendations: avoidance of neuroexcitant substances, continuation of treatment with doxycycline for 15 days, and to attend psychiatric, infectious disease, and serology checkups, depending on progress. The most important events of the disease progression are shown in the timeline (Figure 2).

The patient was invited for the first posthospitalization control as an outpatient of the Infectious Disease Clinic on the 44th day. Biochemistry, hematology, and urine laboratory tests showed all results to be in the reference range, and a CSF sample was collected in order to consolidate the diagnosis of LNB. Glucose, protein, and albumin levels were all normal, and no lymphomonocytic pleocytosis was present. The repeated Borrelia enzyme-linked immunosorbent assay tests of paired serum and CSF samples confirmed serum IgM and IgG positivity, along with CSF IgG positivity. This time, the CSF Borrelia IgM and IgG immunoblots could be repeated with low dilutions (1:2 for IgM and 1:4 for $\mathrm{IgG}$ ) and were positive for IgG (p100, VIsE, B. afzelii $\mathrm{p} 18$, and lesser reactions for p58 and p39), but not in the case of IgM. The patient was monitored for 24 hours and discharged with recommendation for antidepressant and anxiolytic treatment. On the occasion of the second control visit on the 122nd day, there was no change in his serological status according to the Western blot analysis. The patient showed a good general condition, coherent thinking and speaking, with the only complaint of right arm paresthesia, but without any other pathological findings on clinical examination. In summary, the outcome of the disease was very favorable, the patient being able even to drive his car. He was discharged with low-dose alprazolam and mirtazapine maintenance treatment. 


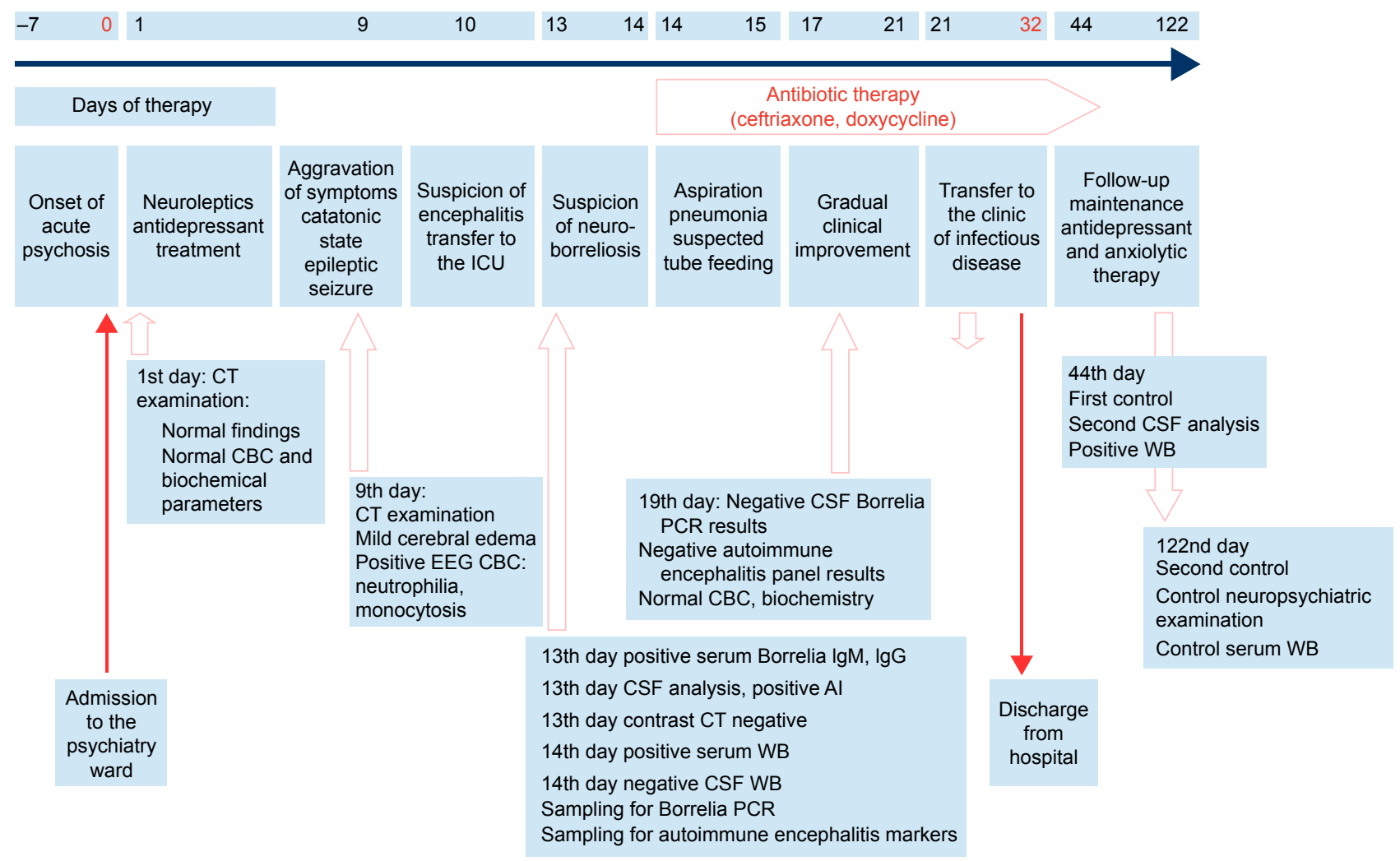

Figure 2 Timeline graph of the main events in our patient's disease course.

Abbreviations: ICU, intensive care unit; Ig, immunoglobulin; CSF, cerebrospinal fluid; CT, computed tomography; PCR, polychromase chain reaction; CBC, complete blood count; WB, Western blot test.

\section{Discussion}

The intrathecal presence of Borrelia-specific antibodies is the immunological hallmark of neuroborreliosis. The diagnosis of acute neuroborreliosis is partially clinical, being supported by the following CSF findings: elevated leukocyte count, bloodbrain barrier dysfunction, elevated IgM levels, and AI for IgM and IgG. In a study performed on an LNB cohort, AI for IgM and $\operatorname{IgG}$ was elevated in $\sim 70 \%$ of the patients, being more prominent in the subgroups presenting polyradiculoneuritis and isolated facial palsy than in the meningitis subgroup. ${ }^{13}$

Very few cases with an organic psychotic syndrome and lacking peripheral neurologic signs of LNB are mentioned in literature. ${ }^{4,7}$ In a similar case, a 31-year-old woman with acute psychosis and high-titer Borrelia seropositivity, but no history of tick-bite, reacted promptly to a combined ceftriaxone and doxycycline treatment. ${ }^{6}$ Another patient, with an initially untreated meningopolyradiculitis, evolved into an acute schizophrenia-like psychosis, and recovered after 1 week of ceftriaxone treatment. ${ }^{11}$ However, overt or putative LNB is sometimes difficult to differentiate from primary psychiatric disorders, such as depression or schizophrenialike psychosis. ${ }^{14}$
Chronic or late-phase neuroborreliosis is characterized by the presence of IgG type antibodies and a positive CSF/ serum IgG AI. The risk of negative serological and intrathecal findings is low at the time of initial clinical presentation. Kaiser ${ }^{15}$ reported a $49 \%$ prevalence of serum, $35 \%$ of CSF IgM type, $81 \%$ of serum, and $90 \%$ of CSF IgG type antibodies in blot and enzyme immunoassay results from patients with acute or chronic neuroborreliosis, applying antigens from three different Borrelia strains: B. burgdorferi, B. afzelii, and B. garinii.

According to Ljostad et al, ${ }^{16}$ the diagnostic sensitivity of $\mathrm{AI}$ is $100 \%$ when symptoms have a duration of at least 6 weeks, but it is not optimal within the first 6 weeks of progression. In patients with a negative pretreatment, AI generally remains with a negative finding at follow-up.

It should be noted that our patient presented a serum antibody profile specific for different Borrelia strains (p39, p100 - B. afzelii, p58 - B. garinii, VIsE, p18-mixtures of strains). He also possessed an increased IgG AI and, at the second CSF testing, Borrelia antibody-specific Western blot proved the presence of anti-p100, anti-p18, and antiVIsE IgG type antibodies. In the absence of pleocytosis and 
typical peripheral neurological signs - but with the presence of psychosis, cognitive disorders, and a positive IgG $\mathrm{AI}$ - the suspicion of neuroborreliosis is real, but according to a recently proposed diagnostic algorithm, it indicates a previous central nervous system infection, not an active disease stage. ${ }^{17}$ However, in our case, the clinically successful antibiotic therapy is supportive for an ongoing, active Lyme disease with a putative neurological involvement.

In Europe, the most common neuroborreliosis-causing species is B. garinii. ${ }^{18,19}$ Clinical diagnosis is more specific in the case of $B$. garinii infection, but difficult and often missed when B. afzelii is the causative agent. In Europe, associated inflammatory response is more commonly met: meningitis, meningoradiculitis (Bannwarth syndrome), peripheral neuropathy, and (more rarely) encephalitis and encephalomyelitis being described. ${ }^{20}$ Our patient possessed IgG type antibodies against $B$. afzelii (p100, p18, p39) and B. garinii (p58) and common epitopes (VIsE) both in serum and CSF, and also IgM type antibodies against Osp $\mathrm{C}$ of B. burgdorferi, B. afzelii, B. garinii, and B. spielmanii in serum: a condition which raises the possibility of repeated infections with different Borrelia species. The probability of reinfection is the subject of intensive debate, ${ }^{21,22}$ but in our case, where the professional exposure was real, it has to be taken into consideration.

In a previous study, tests applying recombinant Borrelia antigens were shown to have the best sensitivity and specificity. ${ }^{23}$ The frequency of antibody specificities were described as follows: VisE (100\%), Osp C (79\%), p18 (52\%), and p58 (50\%). In this study, Osp C positive samples were also characteristically reactive for two or more Borrelia species.

Taking into consideration the suggested clinical picture, anti-NMDAR encephalitis had to be excluded as an important condition for differential diagnosis.

Anti-NMDAR antibodies were also observed in demyelinating encephalomyelitis and neuromyelitis optica disorders. ${ }^{24}$ Anti-NMDAR encephalitis is often misclassified as catatonic schizophrenia, but neurometabolic investigations may elucidate the hypo-glutaminergic status. ${ }^{25}$ Availability of NMDAR testing in different Eastern-European countries and medical centers is highly variable. Our patient presented negative anti-NMDAR serum and CSF tests, and also an absence of other neuroimmune autoantibodies (CASP2, GABA-2R, AMPA1, AMPA2, and LGI1).

CSF analysis in anti-NMDAR encephalitis presents variable findings. Lymphocytic pleocytosis is characteristic for $80 \%$, with total protein increase in $\sim 33 \%$ and oligoclonal bands in $25 \%$ of the cases. ${ }^{24}$ These conditions were lacking in our case. In the absence of prodromal signs, a typical clinical picture, CSF pleocytosis, and body temperature changes, infectious encephalitis was not considered as an underlying pathology.

Acute encephalopathy with catatonic manifestation of chronic neuroborreliosis has very rarely been documented before. ${ }^{26}$ Autoimmune reactions based on molecular mimicry and Borrelia glycolipid induction of the proinflammatory cytokines interleukin (IL)-6, IL-8, and IL-12 as well as chemokines, such as chemokine (C-X-C motif) ligand 12 and 13 (CXCL12 and CXCL13), may be responsible for these scenarios. ${ }^{27}$

Prolonged, up to 26-52-week antibiotic therapy, was proposed to improve cognitive functions, fatigue, and myalgia in patients with neuroborreliosis. ${ }^{28}$ However, in this case, antibiotic therapy of conventional duration applied in conformity with the European Concerted Action on Lyme Borreliosis recommendations proved to be efficient.

\section{Conclusion}

When occupational risk factors emerge in patients with acute neuropsychiatric disease, the possibility of Lyme neuroborreliosis should be considered even in the absence of a tickbite history and lack of previous erythema migrans. Taking into consideration the limited diagnostic value of serology and molecular biology, and the limited availability of CSF for repeated analysis, a differential diagnosis approach in the suspicion of neuroborreliosis sometimes has to rely on elimination strategies. Analyzing the possibilities in our patient - based on the clinical picture and laboratory findings - we interpret the case as either an LNB with atypical neuropsychiatric appearance or LNB associated with a neuropsychiatric disease onset. It should be noted that this classification is supported by the significant and sustainable clinical improvement obtained by conventional antibiotic therapy.

\section{Acknowledgments}

We thank Anett Zsófia Gyöngyösi for technical assistance; Dr Maria Gorea and Dr Szabolcs Szatmári for electroencephalogram interpretation; Virág-Lilla Rácz and David Jeremy Speight for their generous help in language editing; and Tibor Kolozsvári for his kind logistic support.

\section{Author contributions}

EEN analyzed and interpreted the laboratory data and drafted the article. AR provided a substantial proportion of clinical data and contributed to the study concept. EU, GT, and TB 
contributed to data acquisition, data analysis, and study concept. EH and AG performed the critical revision of the manuscript. IK-Z elaborated the concept of the case presentation and provided clinical data. All authors contributed toward data acquisition, drafting and critically revising the paper and agree to be accountable for all aspects of the work.

\section{Disclosure}

The authors report no conflicts of interest in this work.

\section{References}

1. Miklossy J. Chronic or late lyme neuroborreliosis: analysis of evidence compared to chronic or late neurosyphilis. Open Neurol J. 2012;6: $146-157$.

2. Mygland A, Ljostad U, Fingerle V, Rupprecht T, Schmutzhard E, Steiner I; European Federation of Neurological Societies. EFNS guidelines on the diagnosis and management of European Lyme neuroborreliosis. Eur J Neurol. 2010;17(1):8-16.

3. Oksi J, Kalimo H, Marttila RJ, et al. Inflammatory brain changes in Lyme borreliosis - a report on three patients and review of literature. Brain. 1996;119:2143-2154.

4. Reess J, Mauch E, Kornhuber HH. Subacute organic psychosyndrome as a clinical manifestation of infection with stage II Borrelia burgdorferi without further neurologic manifestations. Nervenarzt. 1991; 62(8):514-515.

5. van den Bergen HA, Smith JP, van der Zwan A. Lyme psychosis. Ned Tijdschr Geneeskd. 1993;137(41):2098-2100.

6. Csaszar T, Patakfalvi A. Differential diagnostic problems in Lyme disease (Borrelia infection resulting in acute exogenous psychosis). Orv Hetil. 1994;135(41):2269-2271.

7. Markeljevic J, Sarac H, Rados M. Tremor, seizures and psychosis as presenting symptoms in a patient with chronic lyme neuroborreliosis (Lnb). Coll Antropol. 2011;35:313-318.

8. Stricker RB, Winger EE. Musical hallucinations in patients with Lyme disease. South Med J. 2003;96(7):711-715.

9. Fallon BA, Nields JA, Parsons B, Liebowitz MR, Klein DF. Psychiatric manifestations of Lyme borreliosis. J Clin Psychiatry. 1993;54(7): 263-268.

10. Pennekamp A, Jaques M. Chronic neuroborreliosis with gait ataxia and cognitive disorders. Praxis. 1997;86(20):867-869.

11. Roelcke U, Barnett W, Wildersmith E, Sigmund D, Hacke W. Untreated neuroborreliosis: Bannwarth's syndrome evolving into acute schizophrenia-like psychosis. A case report. J Neurol. 1992;239(3):129-131.
12. Binalsheikh IM, Griesemer D, Wang S, Alvarez-Altaleff R. Lyme neuroborreliosis presenting as Alice in Wonderland syndrome. Pediatr Neurol. 2012;46(3):185-186.

13. Djukic M, Schmidt-Samoa C, Lange P, et al. Cerebrospinal fluid findings in adults with acute Lyme neuroborreliosis. J Neurol. 2012; 259(4):630-636.

14. Bar KJ, Jochum T, Hager F, Meissner W, Sauer H. Painful hallucinations and somatic delusions in a patient with the possible diagnosis of neuroborreliosis. Clin J Pain. 2005;21(4):362-363.

15. Kaiser R. False-negative serology in patients with neuroborreliosis and the value of employing of different borrelial strains in serological assays. J Med Microbiol. 2000;49(10):911-915.

16. Ljostad U, Skarpaas T, Mygland A. Clinical usefulness of intrathecal antibody testing in acute Lyme neuroborreliosis. Eur J Neurol. 2007; 14(8):873-876.

17. Koedel U, Fingerle V, Pfister H-W. Lyme neuroborreliosis-epidemiology, diagnosis and management. Nat Rev Neurol. 2015;11(8):446-456.

18. Stanek G, Wormser GP, Gray J, Strle F. Lyme borreliosis. Lancet. 2012;379(9814):461-473

19. Hildenbrand P, Craven DE, Jones R, Nemeskal P. Lyme neuroborreliosis: manifestations of a rapidly emerging zoonosis. AJNR Am JNeuroradiol. 2009;30(6):1079-1087.

20. Fallon BA, Levin ES, Schweitzer PJ, Hardesty D. Inflammation and central nervous system Lyme disease. Neurobiol Dis. 2010;37(3): 534-541.

21. Nadelman RB, Wormser GP. Reinfection in patients with Lyme disease. Clin Infect Dis. 2007;45(8):1032-1038.

22. Donta ST. Reinfection versus relapse in Lyme disease. $N$ Engl J Med. 2013;368(11):1063-1063.

23. Henningsson AJ, Christiansson M, Tjernberg I, Lofgren S, Matussek A. Laboratory diagnosis of Lyme neuroborreliosis: a comparison of three CSF anti-Borrelia antibody assays. Eur J Clin Microbiol Infect Dis. 2014;33(5):797-803.

24. Dalmau J, Lancaster E, Martinez-Hernandez E, Rosenfeld MR, BaliceGordon R. Clinical experience and laboratory investigations in patients with anti-NMDAR encephalitis. Lancet Neurol. 2011;10(1):63-74.

25. Endres D, Perlov E, Stich O, et al. Hypoglutamatergic state is associated with reduced cerebral glucose metabolism in anti-NMDA receptor encephalitis: a case report. Bmc Psychiatry. 2015;15:186.

26. Pfister HW, Preacmursic V, Wilske B, et al. Catatonic syndrome in acute severe encephalitis due to Borrelia burgdorferi infection. Neurology. 1993;43(2):433-435.

27. Bransfield RC. The psychoimmunology of lyme/tick-borne diseases and its association with neuropsychiatric symptoms. Open Neurol J 2012;6:88-93.

28. Stricker RB, Delong AK, Green CL, Savely VR, Chamallas SN, Johnson L. Benefit of intravenous antibiotic therapy in patients referred for treatment of neurologic Lyme disease. Int J Gen Med. 2011;4:639-646.
Neuropsychiatric Disease and Treatment

\section{Publish your work in this journal}

Neuropsychiatric Disease and Treatment is an international, peerreviewed journal of clinical therapeutics and pharmacology focusing on concise rapid reporting of clinical or pre-clinical studies on a range of neuropsychiatric and neurological disorders. This journa is indexed on PubMed Central, the 'PsycINFO' database and CAS,

\section{Dovepress}

and is the official journal of The International Neuropsychiatric Association (INA). The manuscript management system is completely online and includes a very quick and fair peer-review system, which is all easy to use. Visit http://www.dovepress.com/testimonials.php to read real quotes from published authors. 